\title{
Seamless Location Measuring System with Wifi Beacon Utilized and GPS Receiver based Systems in Both of Indoor and Outdoor Location Measurements
}

\author{
Kohei Arai 1 \\ Graduate School of Science and Engineering \\ Saga University \\ Saga City, Japan
}

\begin{abstract}
A seamless location measuring system with WiFi beacon utilized and GPS receiver based systems in both of indoor and outdoor location measurements is proposed. Through the experiments in both of indoor and outdoor, it is found that location measurement accuracy is around 2-3 meters for the locations which are designated in both of indoor and outdoor.
\end{abstract}

Keywords-GPS receiver; WiFi beacon; seamless location estimation

\section{INTRODUCTION}

GPS based location estimation is quite popular. Mobile device, smart-phone, i-phone are utilizing GPS receivers for location estimation. It, however, does not work for indoor environments because of the fact that GPS satellite signals cannot be received in indoor environments. The location estimation accuracy of the GPS receiver based method is not so high. Furthermore, the accuracy of GPS based location estimation depends on many factors such as weather condition, the number of the acquired GPS satellites, circumstances (surrounding buildings, mountains, etc.), etc. On the other hands, WiFi-beacon can be used for location measurements in both indoor and outdoor environments. The location estimation accuracy depends on the situation of WiFi access points. Therefore, in general, $\mathrm{WiFi}$ beacon utilized location estimation accuracy is not good. It, however, still is somewhat useful for location estimation if the location accuracy requirement is not high. Therefore, $\mathrm{WiFi}$ beacon receiver based location estimation method is proposed here for both indoor and outdoor situations.

Bose and Heng classified WiFi-based positioning methods into Cell Identity (Cell-ID), Time of Arrival (TOA), Time Difference of Arrival (TDOA), Angle of Arrival (AOA), and signal strength categories [1]-[9]. Cell Identity (Cell-ID) is a basic wireless positioning system solution. It matches the target's position with its connection to an Access Point (AP). It does not require complex operations such as time synchronization and multiple APs. However, its low positional accuracy is the pitfall of its simplicity. Time of Arrival (TOA) measures a distance using the travel time of a radio signal from a transmitter to a receiver. Its application requires time synchronization of the transmitter and receiver, which is difficult to achieve for close ranges. To overcome the problem, Time Difference of Arrival (TDOA) was developed, which utilizes the time difference between receiver and two or more receivers. That is to say, whereas TOA requires time synchronization of transmitters and receivers, TDOA needs just synchronization between receivers. Angle of Arrival (AOA) determines the position of a receiver by measuring the angle to it from a transmitter. An AP must use smart antennas and be capable of mounting them under static conditions.

Signal Strength based technique uses the signal attenuation property of the radio wave Received Signal Strength Indication (RSSI) to measure the distance from a receiver to transmitter using the distance-to-signal-strength relationship. One common approach of RSSI-based system is fingerprint approach, which entails two phases: a training phase and a tracking phase. In the training phase, the received signal strength information is filtered, interpolated, and eventually stored in a database as sample points. In the tracking phase, the position is determined by comparison with the received signal strength sample points stored in the database [10]. The accuracy of this system is a function of the sample points' sampling space, an estimation method and the structure of the database. However, such a method requires the time consuming on survey procedure or calibration process.

In order to find patients in hospitals, victims in group homes, etc., GPS receivers and $\mathrm{WiFi}$ beacon receivers in smart-phone, i-phone and tablet terminals are used in the proposed system. The location of $\mathrm{WiFi}$ access points the designated hospitals and the supposed group homes are known. Also, the specific location of the hospitals and group homes, for instance, the middle of the entrance door, is known. Therefore, the locations of the patients and the victims are estimated in both of indoor and outdoor situations in seamless basis. When they are in hospitals or group homes, their locations are estimated with $\mathrm{WiFi}$ beacon receivers while their location is estimated with $\mathrm{WiFi}$ beacon receiver and GPS receivers when they are in outside of hospitals or group homes with an accuracy of a couple of meters ${ }^{1}$. WiFi beacon based location estimation is helpful to improve GPS based location accuracy $^{2}$. Also, location estimation can be done with WiFi

\footnotetext{
${ }^{1}$ http://wiki.openstreetmap.org/wiki/Accuracy_of_GPS_data

${ }^{2} \mathrm{http}: / /$ www.quora.com/Why-is-location-accuracy-improved-when-wi-fi-isenabled
} 
beacon receiver in smart-phone, i-phone, and tablet terminal ${ }^{3}$. The WiFi beacon based location estimation accuracy around a couple of meters in an indoor situation [11]-[12].

The location estimation method proposed here is to use both GPS based method with improvement by WiFi beacon based method (it is referred to GPS based method hereafter) in outdoor situations and WiFi beacon based method in indoor situations as well as a calibration of estimated locations at the specific location of hospitals or group homes. Because of the locations of specific positions of hospitals or group homes are known, a calibration can be done through a comparison of the estimated locations of the designated specific positions between GPS based and WiFi beacon based methods. Thus the locations of the patients in hospitals and / or victims in group homes are estimated in seamless basis. Through experiments, it is found that the proposed method does work for seamless location estimation with an acceptable accuracy, 2-3 meters for finding the patients and the victims when they are out of hospitals or group homes when a disaster occurs.

The next section describes the proposed seamless basis location estimation method followed by some experiments. Then conclusion is described together with some discussions.

\section{THE PROPOSED METHOD AND SYSTEM}

The location estimation method proposed here is to use both GPS based method and WiFi beacon based method as well as a calibration of estimated locations at the specific location of hospitals or group homes. Because of the location of specific position of hospitals or group homes is known, a calibration can be done through a comparison of the estimated locations of the designated specific positions between GPS based and WiFi beacon based methods. Thus the locations of the patients in hospitals and / or victims in group homes are estimated in seamless basis.

Fig.1 shows flow chart of the proposed location estimation method. The location of smart-phone i-phone is estimated with GSP and $\mathrm{WiFi}$ beacon receivers equipped in the smartphone and i-phone. The estimated locations are compared to the location of the designated known position in a prior to the location estimation such as the center of the entrance of the hospital or the group home. Then calibration of location estimation is done with the difference between the designated location and the estimated location with GPS and WiFi beacon. Thus the estimated location is calibrated. Repeat the calibration and location estimation is repeated.

The estimated locations are expressed with ISO 19155 of Place Identifier: PI. Example of the PI expression is shown below,

$<$ allpi>

$<$ placeidentifier $>$

<name>Kasasagi-Kaikan</name>

<latitude>33.24149339</latitude>

<longitude>130.28919659</longitude>

$</$ placeidentifier $>$

\footnotetext{
${ }^{3}$ http://engineeringblog.yelp.com/2012/08/gps-vs-wifi-the-battle-for-locationaccuracy-using-yelp-check-ins.html
}

$<$ placeidentifier $>$

<name>Faculty Bldg. No.6</name>

$<$ latitude $>33.24139665<$ /latitude $>$

$<$ longitude $>130.28864292<$ /longitude $>$

$</$ placeidentifier $>$

$<$ placeidentifier $>$

<name>faculty Bldg. No.7</name>

$<$ latitude $>33.24118506<$ /latitude $>$

$<$ longitude $>130.28842333<$ /longitude $>$

$</$ placeidentifier $>$

$</$ allpi $>$

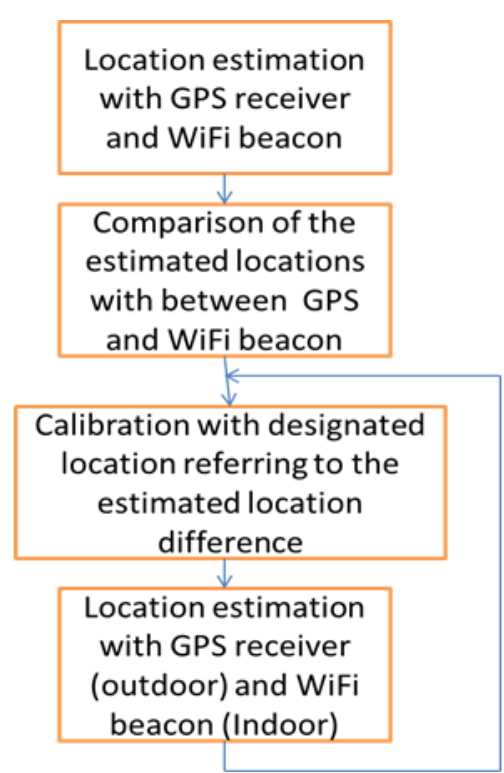

Fig. 1. Flow chart of the proposed location estimation method

The location name is located at the third line followed by latitude and longitude of the location. These name and latitude / longitude are aligned sequentially. PI expression is based on XML. Therefore, other information which is related to the location can be attached with a tag or tags. For instance, phone number, address, and the others. Therefore, these are referred crossly each other. Thus users can retrieve the location with the name, the phone number, the address, and the latitude / longitude.

\section{EXPERIMENTS}

\section{A. Location estimation accuracy with GPS receiver based method (Improvement by WiFi beacon receiver based method) in outdoor situation}

Although location estimation accuracy of the GPS receivers is well reported, there are a few reports on location estimation accuracy of $\mathrm{WiFi}$ beacon receivers. Therefore, the following experiments are conducted at the road situated at the prefectural border between Fukuoka and Saga, Japan in night time. Test site on the map is shown in Fig. 2 while the photo of the test site in day time is shown in Fig.3. At the test site on the Sazanka road, WiFi access points and mobile devices are set up as are shown in Fig.4.

In order to avoid external noises, location estimation accuracy is measured in night time. The distance between two 
locations, A and B is $10 \mathrm{~m}$. Distance from A and B is measured with $1 \mathrm{~m}$ step. Received signal strength is varied with the distance between the location $\mathrm{A}$ and the location apart from A with $1 \mathrm{~m}$ step. Therefore distance between both locations can be estimated.

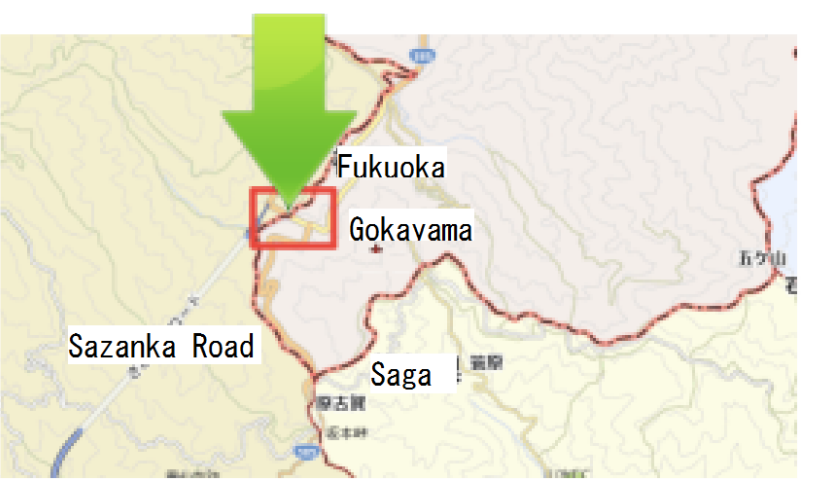

Fig. 2. Test site location on topographic map

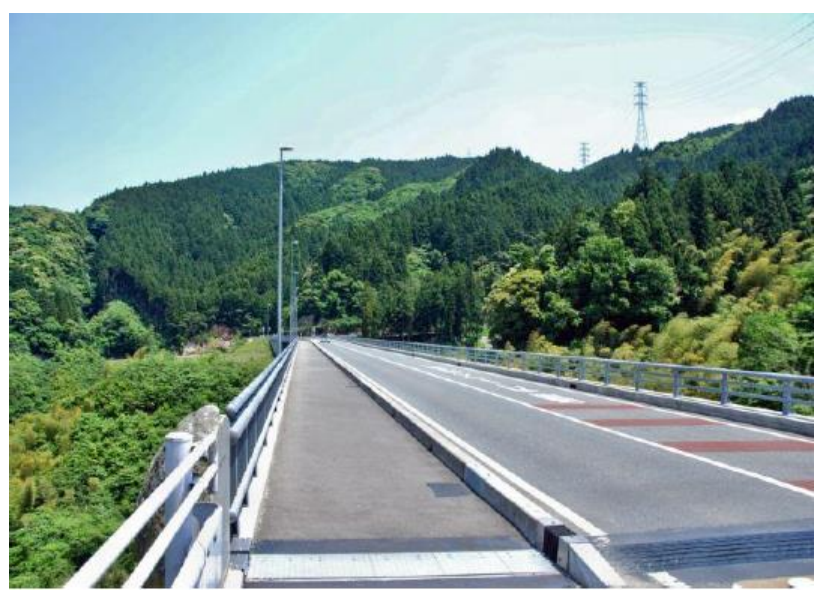

Fig. 3. Day time photo of the test site situated at the prefectural border between Fukuoka and Saga, Japan

Received signal strength in unit of $\mathrm{dBm}$ is shown in Table 1 while the estimated distance between both locations is shown in Table 2, respectively.

As the results of the experiments, it is found that location estimation error of the method of location estimation with WiFi beacon is around $5(\%)$.

TABLE I. RECEIVED SignAl AT THE DIFFERENT LOCATIONS, A AND B

Distance(m) Signal_Level_from_A(dBm) Signal_Level_from_B(dBm)

\begin{tabular}{|c|c|c|}
\hline 0 & -25 & -58 \\
\hline 1 & -31.5 & -57 \\
\hline 2 & -35.5 & -56.5 \\
\hline 3 & -39 & -55 \\
\hline 4 & -43 & -52 \\
\hline 5 & -45 & -50 \\
\hline 6 & $-47 ; 5$ & -47 \\
\hline 7 & -49 & -45 \\
\hline 8 & -51.5 & -48 \\
\hline 9 & -54 & -38 \\
\hline 10 & -57.5 & -27 \\
\hline
\end{tabular}

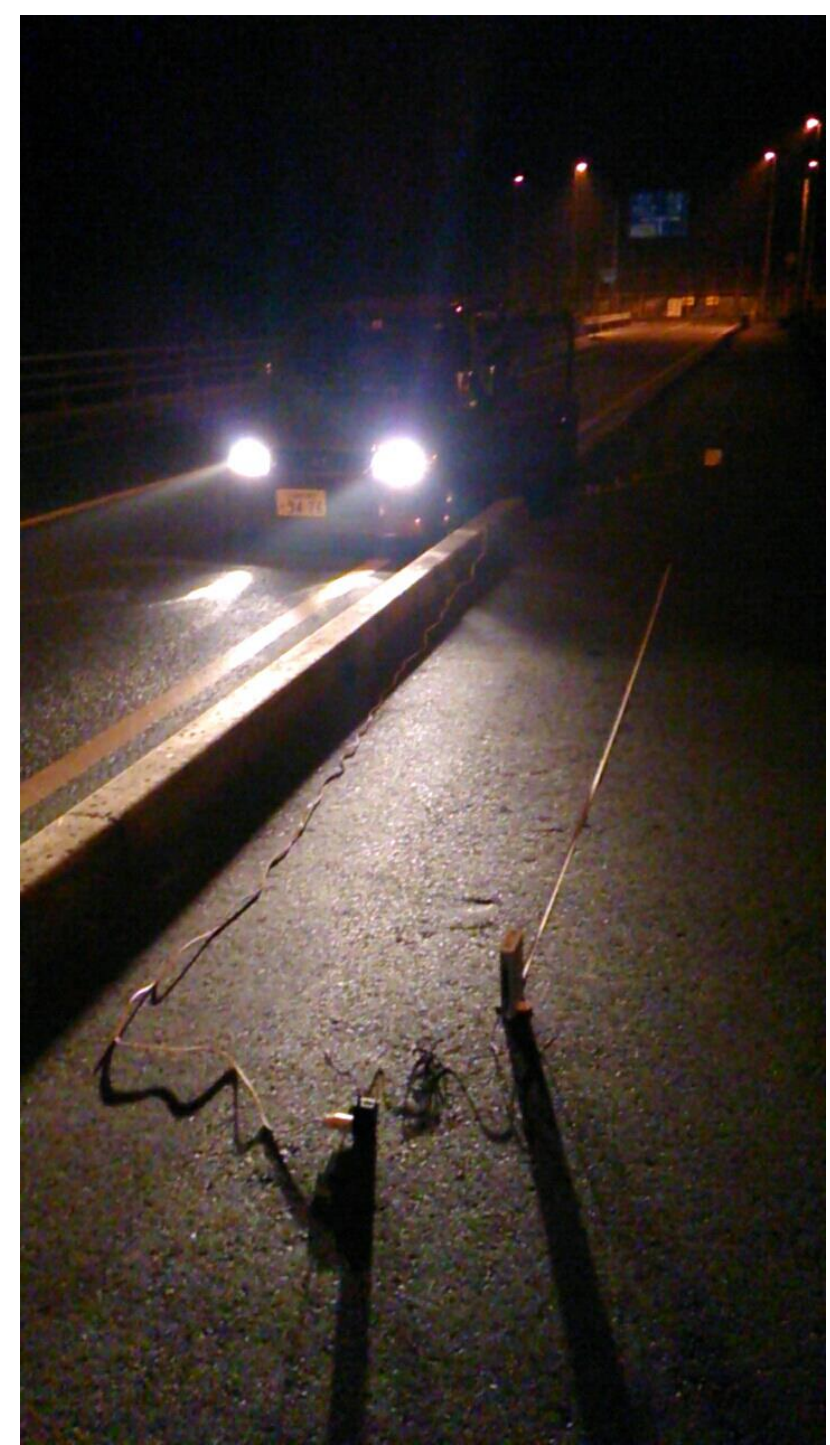

Fig. 4. WiFi access points and mobile device are set up on the Sazanka road of the test site

TABLE II. MEASURED DisTANCE ERRORS

\begin{tabular}{|c|c|c|}
\hline Distance $(\mathrm{m})$ & Calculated_Distance $(\mathrm{m})$ & Error $(\%)$ \\
\hline 0 & -0.28 & 28.0 \\
\hline 1 & 1.06 & 5.66 \\
\hline 2 & 1.89 & -5.5 \\
\hline 3 & 2.8 & -6.67 \\
\hline 4 & 4.15 & 3.61 \\
\hline 5 & 5.0 & 0.0 \\
\hline 6 & 6.16 & 2.6 \\
\hline 7 & 6.84 & -2.29 \\
\hline 8 & 7.62 & -4.99 \\
\hline 9 & 8.89 & -1.22 \\
\hline 10 & 11.2 & 10.7 \\
\hline
\end{tabular}

Location estimation accuracy of the GPS receiver based method is around 3.4 meters in average. Therefore, GPS receiver based method with the improvement by $\mathrm{WiFi}$ receiver based method achieved about 6 times much accurate location estimation. 


\section{B. Location estimation accuracy with WiFi beacon receiver based method in indoor situation}

As described above, wireless channel signal strengths are changeful. Even immovable calibrating, the signal strengths still pulse up and down. Inconstant signal strengths on one position make estimating location difficult and inaccurate. Most research adopts mean value to solve this problem, but I think it's insufficient through inexact location estimating result. Using one mean value to stand for one position's wireless channel information is not enough. I present a Grid Segment Process to make some improvement. Assume one training process gains 100 signal strengths, I divide up these 100 signal strengths into 10 parts, each has 10 signal strengths. Then calculate mean value of each part and store them into radio map to substitute for original one mean value. Now I acquire 10 slices of mean values and have more information to estimate location. I call the divide procedure as Segment Process. Not only in offline calibration phase I do Segment Process, but in online estimation phase I execute it, as will introduce below. The mean value is used for expressing proper wireless channel characteristic. Using Segment Process to obtain more slices of mean values denotes more information to refer to and more accurate location estimation result. The received signal of each access point is converted into its color representative. This system use 3 signals information from 3 different AP's. Each access point has its basic color that different each other. The three AP ( $A P 1, A P 2$, $\&$ AP3) use red, green and blue color respectively. The color map is based on signal strength information get from signal data collection process. The gradation of color is based on the HSL (Hue Saturation Luminance) value where $L$ is a function of signal strength. Assuming $S_{p x}$ is a variable for signal strength (in percentage) for the position of $x$ meter from the initial position. Then the color for the grid of $x$ meter distance is measured by the formula:

\section{$L$-Color $A P 1_{x}=255-\left(\right.$ Hexadecimal $\left.\left(S_{p x}\right) * 128 / 100\right)$}

where Hexadecimal(S) is function for convert the input into its hexadecimal value.

The gradation of color is based on the HSI (Hue Saturation Intensity) value where $\mathrm{L}$ is a function of signal strength.

The fission of the grid is half size and the color is based on the RGB combination of two adjacent colors. Then we will have a new grid in the more detail size as shown in Fig.5. The second stage of grid fusion is possible to create more detail color radio map grid. The accuracy of each color radio-map then investigates to measure the accuracy.

The effectiveness of this method is the interpolation using color grid fusion technique. Initial grid size could setup to a number that low cost on offline training. I start with grid size of 5 meters long with 2.5 widths (the corridor width). The initial color radio-map is created for this grid size that will have the initial error also 5 meters. Then too improve the accurate of the system, the initial map is interpolate in half size to determine the interpolation value (color) of middle point between two grids. Fig. 6 shows the illustration of grid fusion technique. Also Fig.7 shows an example of location estimation result in the color radio-map representation.

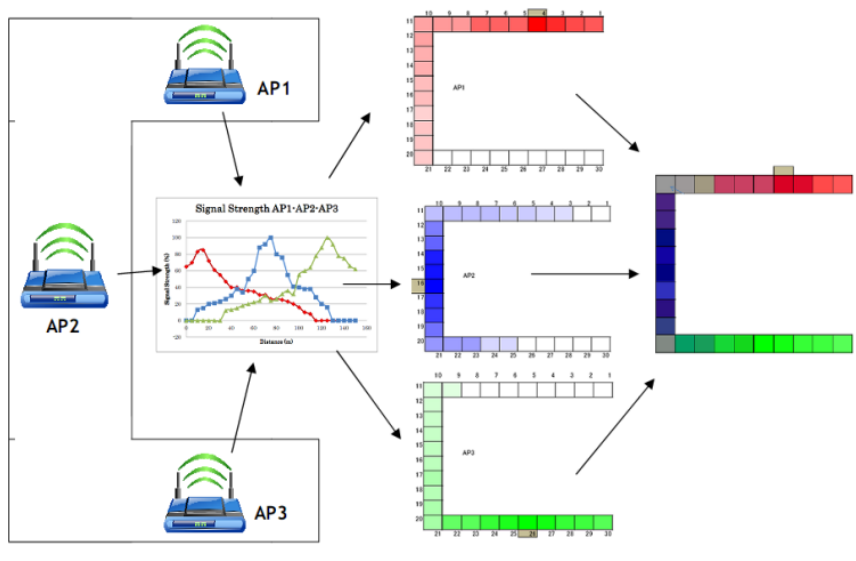

AP signal $\rightarrow \quad$ Signal Graph $\rightarrow \quad$ Color Representation $\rightarrow \quad$ Color Combination

Fig. 5. Diagram flow of Color Radio Map Method
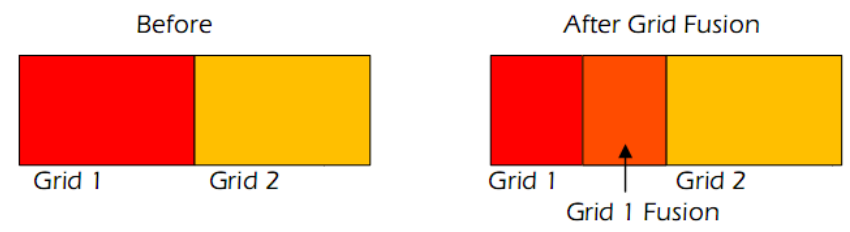

Fig. 6. Grid fusion techniques

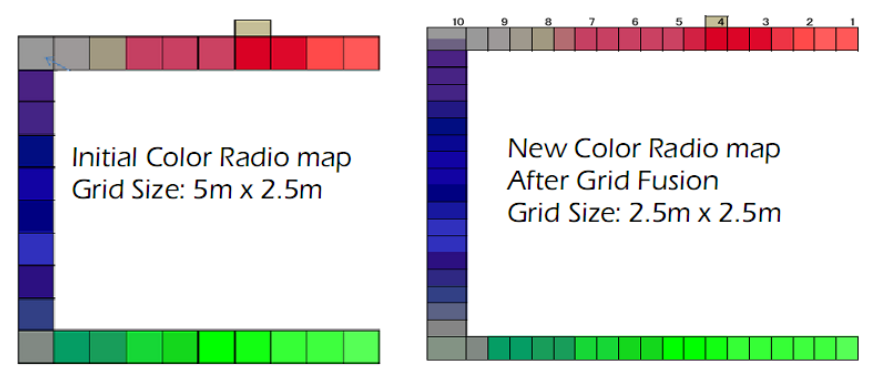

Fig. 7. Example of an estimated result

I performed our experiment in the third floor of the Science and Engineering Faculty Building No.1, Saga University. This building has a layout like show in Fig. 8 with the total dimension in rectangle is $150 \times 3$ meters. The building is equipped with $802.11 \mathrm{~b}$ wireless LAN environment. To form the radio map, the environment was modeled as a space of 30 locations in grid of $5 \times 3$ meters each. The position of AP and initial online tracking position is show in Fig.5.

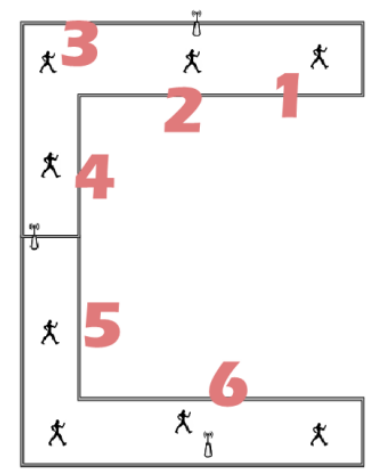

Fig. 8. Floor layout of the Bldg. in the experiment 
As the result of the experiment, it is found that the location estimation accuracy is approximately $2.5 \mathrm{~m}$.

\section{Calibration of estimated location}

Application software is developed which allows location estimation with GPS and WiFi beacon receivers. The estimated location is expressed with PI. Example of the estimated locations with GPS and WiFi beacon is shown in Fig.9. Not only latitude and longitude but also direction of the target location from the current position is displayed onto smart-phone and / or i-phone as shown in Fig.10. The direction can be calculated with the following equation.

$\theta=\operatorname{atan}(2 \beta / \alpha)$

where $\alpha$ and $\beta$ are calculated with the estimated locations.

ISO19155: Place Identifier (PI)

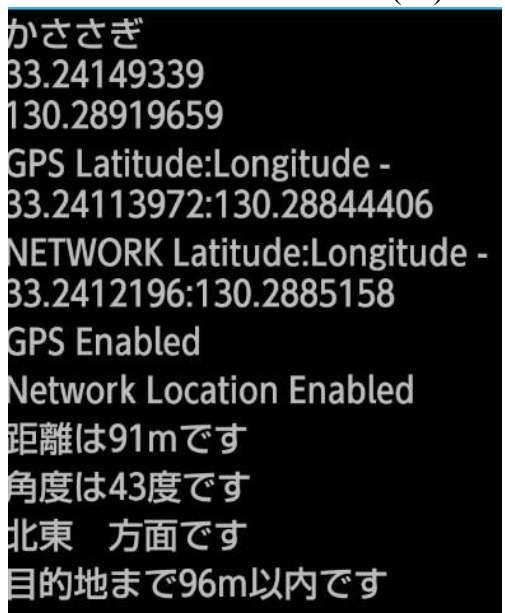

Fig. 9. Example of the estimated locations displayed onto smart-phone or iphone

Example of the estimated and actual location is shown in Fig.11. In comparison of the actual location and the estimated locations with GPS and $\mathrm{WiFi}$ receivers are quite clearly different each other with around 5 meters. Location estimation accuracies of GPS and WiFi beacon based methods depend on circumstances, surrounding building, in this case.

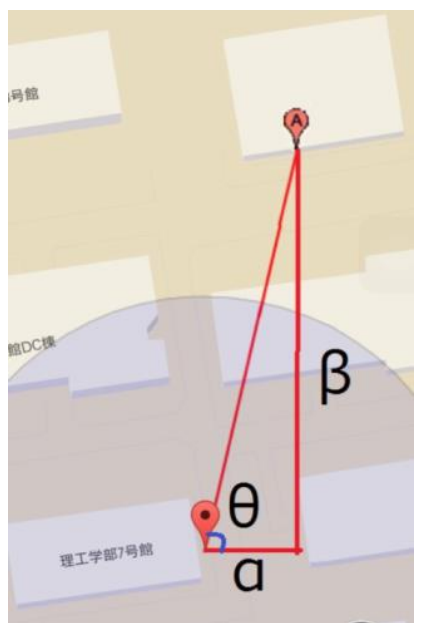

Fig. 10. Estimation of the direction of the target location from the current location
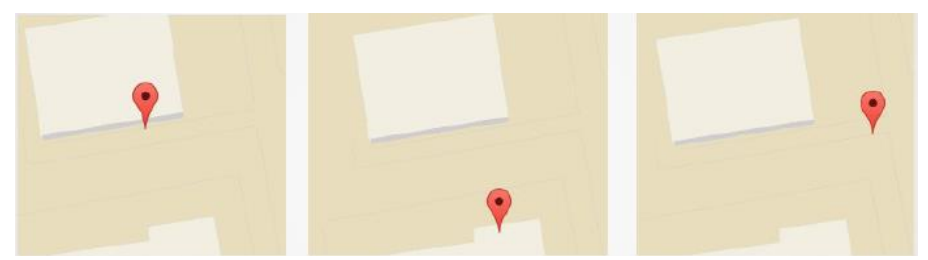

(a)Actual location

(b)GPS

(c) $\mathrm{WiFi}$

Fig. 11. Actual and the estimated locations with GPS and WiFi beacon receivers equipped in smart-phone and i-phone

Using the difference between the actual location and the estimated locations with GPS and $\mathrm{WiFi}$ beacon receivers can be calibrated. Thus the estimated location is switched to GPS based method to $\mathrm{WiFi}$ beacon based method when the patients and the victims are getting into hospitals and or group homes from the outside and vise versa.

\section{CONCLUSION}

A seamless location measuring system with $\mathrm{WiFi}$ beacon utilized and GPS receiver based systems in both of indoor and outdoor location measurements is proposed. Through the experiments in both of indoor and outdoor, it is found that location measurement accuracy is around 2-3 meters for the locations which are designated in both of indoor and outdoor. It is also found that GPS receiver based location estimation method with improvement by using $\mathrm{WiFi}$ beacon based method works well. Approximately 6 times better accuracy is achieved in comparison between GPS based method and the proposed GPS based method with improvement with $\mathrm{WiFi}$ beacon based method.

These GPS and WiFi beacon receivers are equipped in smart-phone and / or i-phone. Therefore, the locations of the patients in hospitals, the victims in group homes can be estimated with 2-3 meters of accuracy if they have the smartphone and / or i-phone with the developed application software.

\section{ACKNOWLEDGMENT}

The authors would like to thank Mr. Kenji Egashira, Dr. Herman Tolle of Arai's laboratory members for their useful comments and suggestions during this research works.

\section{REFERENCES}

[1] P. Bahl, V.N. Padmanabhan, RADAR: an in-building RF-based user location and tracking system, INFOCOM 2000. 19th Annual Joint Conference of the IEEE Computer and Communications Societies, Proceedings IEEE 2 (2000) 775-784 vol.772.

[2] A. Bose, F. Chuan Heng, A Practical Path Loss Model for Indoor WiFi Positioning Enhancement, Information, Communications \& Signal Processing, $20076^{\text {th }}$ International Conference, 2007, pp. 1-5.

[3] Y. Chen, X. He, Contribution of pseudolite observations to GPS precise surveys, KSCE Journal of Civil Engineering 12 (1) (2008) 31-36.

[4] Y.K. Cho, J.-H. Youn, N. Pham, Performance tests for wireless real-time localization systems to improve mobile robot navigation in various indoor environments, in: C. Balaguer, M. Abderrahim (Eds.), Robotics and Automation in Construction, InTech Education and Publishing, 2008, pp. 355-372.

[5] Y. Fukuju,M. Minami, H.Morikawa, T. Aoyama, DOLPHIN: An Autonomous Indoor Positioning System in Ubiquitous Computing Environment, Proceedings of the IEEE Workshop on Software Technologies for Future Embedded Systems, IEEE Computer Society, 2003. 
[6] S. Han, J. Kim, C.-H. Park, H.-C. Yoon, J. Heo, Optimal detection range of RFID tag for RFID-based positioning system using the k-NN algorithm, Sensors 9 (6) (2009) 4543-4558.

[7] C. Hu, W. Chen, Y. Chen, D. Liu, Adaptive Kalman filtering for vehicle navigation, Journal of Global Positioning Systems 2 (1) (2003) 6.

[8] J. Yim, C. Park, J. Joo, S. Jeong, Extended Kalman filter for wireless LAN based indoor positioning, Decision Support System 45 (4) (2008) 960-971.

[9] W.-S. Jang, M.J. Skibniewski, A wireless network system for automated tracking of construction materials on project sites, Journal of Civil Engineering and Management 14 (1) (2008) 9.

[10] H.M. Khoury, V.R. Kamat, Evaluation of position tracking technologies for user localization in indoor construction environments, Automation in Construction 18 (4) (2009) 444-457.

[11] Arai, K., Tolle Herman, Akihiro Serita, Mobile Devices Based 3D Image Display Depending on Users' Actions and Movements, International Journal of Advanced Research in Artificial Intelligence, 2, 6, 77-83, 2013.
[12] Kohei Arai, Herman Tolle, Color radio-map interpolation for efficient fingerprint WiFi-based indoor location estimation, International Journal of Advanced Research in Artificial Intelligence, 2, 3, 10-15, 2013

\section{AUTHORS PROFILE}

Kohei Aarai He received BS, MS and PhD degrees in 1972, 1974 and 1982, respectively. He was with The Institute for Industrial Science and Technology of the University of Tokyo from April 1974 to December 1978 and also was with National Space Development Agency of Japan from January, 1979 to March, 1990. During from 1985 to 1987, he was with Canada Centre for Remote Sensing as a Post Doctoral Fellow of National Science and Engineering Research Council of Canada. He moved to Saga University as a Professor in Department of Information Science on April 1990 $\mathrm{He}$ was a councilor for the Aeronautics and Space related to the Technology Committee of the Ministry of Science and Technology during from 1998 to 2000. He was a councilor of Saga University for 2002 and 2003. He also was an executive councilor for the Remote Sensing Society of Japan for 2003 to 2005. He is an Adjunct Professor of University of Arizona, USA since 1998. $\mathrm{He}$ also is Vice Chairman of the Commission-A of ICSU/COSPAR since 2008. He wrote 33 books and published 500 journal papers. 